\title{
Hair's Zinc Level on Androgenic Alopecia
}

\author{
Nurul Rumila Roem ${ }^{1}$, Farida Tabri ${ }^{1}$, Nurelly N. Waspodo ${ }^{1}$, Ilhamjaya Patellongi ${ }^{2}$, \\ Agussalim Bukhari ${ }^{3}$, Nursiah La Nafie ${ }^{4}$ \\ ${ }^{1}$ Department of Dermatology and Venereology, Medical Faculty, Hasanuddin University, Makassar, Indonesia \\ ${ }^{2}$ Department of Biostatistic, Faculty of Public Health, Hasanuddin University, Makassar, Indonesia \\ ${ }^{3}$ Department of Clinical Nutrition, Medical Faculty, Hasanuddin University, Makassar, Indonesia \\ ${ }^{4}$ Department of Chemical, Mathematics and Sciences Faculty, Hasanuddin University, Makassar, Indonesia
}

\section{Email address:}

noeroem258@gmail.com (N. R. Roem)

\section{To cite this article:}

Nurul Rumila Roem, Farida Tabri, Nurelly N. Waspodo, Ilhamjaya Patellongi, Agussalim Bukhari, Nursiah La Nafie. Hair's Zinc Level on Androgenic Alopecia. American Journal of Clinical and Experimental Medicine. Vol. 4, No. 5, 2016, pp. 129-133.

doi: 10.11648/j.ajcem.20160405.13

Received: July 19, 2016; Accepted: July 30, 2016; Published: August 25, 2016

\begin{abstract}
Androgenic alopecia is characterized by progressive loss of hair from the scalp. This research aimed to determine the hair and blood zinc levels in men with androgenic alopecia. The research was conducted in the Departement of Dermatovenereology of Dr. Wahidin Sudirohusodo Hospital, Makassar and the Center for Health Laboratory, Makassar, using the observational research method. The samples comprised 21 males with androgenic alopecia and 11 control samples without androgenic alopecia. The hair and blood of the samples were analyzed using the atomic absorption spectrophotometer receipts. The results showed that there was significant difference $(p<0.05)$ between the levels of androgenic alopecia hair zinc and control. On blood zinc levels found no significant differences between androgenic alopecia and control. Hair zinc levels had a significant relationship with the degree of Hamilton, where the lower the levels of zinc in the hair androgenic alopecia, the greater the degree of Hamilton. However, things are different, between blood zinc levels in hair zinc levels and degrees of Hamilton that there is no significant difference. In this study also found between age and degree of Hamilton, where the increasing age, the greater the degree of Hamilton.
\end{abstract}

Keywords: Androgenic Alopecia, Degree of Hamilton, Zinc

\section{Introduction}

Androgenic alopecia or male pattern hair loss is a common progressive hair loss that occurs due to the influence of genetic predisposition and androgens on hair follicles. Although the pattern of hair loss in women are different from men, but female pattern alopecia is also called androgenic alopecia because the characteristics of hair loss similar in both gender, it characterized by shortening the anagen phase, elongation the telogen phase, and downsizing the hair follicles that lead to hair shaft grows thinner in each cycle. Hair loss usually begins in the 20 s or early 30 s with a distinctive pattern which starts from frontal and vertex so the hair line looks backward, leaving hair in the parietal alone. Whereas in women, the pattern of hair loss is more diffuse and it starts from the top of the head. [1]

The incidence rate in males is higher than in women, it is because men have higher level of $5 \alpha$-reductase. $5 \alpha$-reductase plays a role in altering the testosterone hormone into dihydrotestosterone (DHT). DHT then binds to androgen receptors on the hair follicles to form a hormone-receptor complex and activate genes that gradually transform into a large terminal follicles turn to smaller follicles (miniaturization). Hair follicles that undergo miniaturization is a characteristic of androgenic alopecia. According to epidemiology study, the prevalence of androgenic alopecia in male increases with age. In one study showed that nearly $30 \%$ cases occur in 30-years old white men, $50 \%$ at age 50 years and $80 \%$ at age 70 years. The incidence also influenced by race, where white men have higher incidence rate than the Asian, American Indian and African male. It was also found an increasing incidence of benign prostatic hypertrophy $(\mathrm{BPH})$ in men with androgenic alopecia. [2, 3]

The underlying causes of hair loss pattern could not be 
ascertained. In man, male pattern hair loss (MPHL) seems the resulted from a combination of androgen hyperactivity, genetic predisposition for hair loss that is associated with the sensitivity of androgen action as androgen-independent genetic factors. In woman, female pattern hair loss (FPHL) may have a more complex etiology. However, the action of androgen combined with genetic sensitivity appears to play a dominant role in many cases, and these factors can be seen in FPHL. [4, 5]

Previous study by Hamad et al (2010), found that nutritional deficiencies may play a role in increasing hair loss due to the weakening of the hair shaft that causes damage to the hair and slow regrowth. Hair problems caused by nutritional deficiencies can be corrected with a proper diet. Element has many important roles in the growth and health of hair such as copper, iron and zinc. [6]

Zinc is an important micronutrient that plays an important role in the metalloenzyme process of the body. Zinc is involved in the synthesis of proteins and nucleic acids, and plays a role in various metabolic pathways and cellular functions. Zinc is involved in almost every metabolism that occurs in the body, and affects hair growth. It also plays an important functional role in hair follicle cycle. Dealing with hair loss, zinc is a potent inhibitor of the regression of the hair follicle, and accelerate the healing process of the hair follicles. Specifically, zinc deficiency is the main pathogenesis in Enteropathica acrodermatitis, resulting in hair loss. The idea that zinc deficiency may interfere with hair growth has emerged since the 1990s. [7]

Although some studies have reported that zinc deficiency correlates with alopecia areata (AA) and telogen effluvium (TE), there are no studies have examined the relationship between zinc and androgenic alopecia so far. This study is important because there are no studies that examine the relationship between zinc levels with androgenic alopecia. Based on these things, the objective of this study is to determine the hairand blood zinc levels in man with androgenic alopecia.

\section{Material and Methods}

\subsection{Location and Time of Research}

The study was conducted at the Dermatology Department, Dr. Wahidin Sudirohusodo's Hospital and Network Hospitals. Examination conducted in the Laboratory of Chemistry UNHAS carried on September-November, 2015.

\subsection{Design and Research Variables}

The design of this study was observational study. The research variables consist of: the independent variables (zinclevels), the dependent variable (alopecia), confounding variable (5a-reductase), and the control variables (gender and age).

\subsection{Population and Sample}

The study population was all patients with alopecia who visited Cosmetic Dermatology Clinic Division of Health
Sciences Dermatology Dr. Wahidin Sudirohusodo Hospital, Network Hospital in Makassar who aged 20-60 years old. Samples are population who meet the inclusion criteria, willing to participate in the study by signing an informed consent. The selection of study sample is consecutive sampling; it is sampling based on the patient's arrival. The number of samples in this study were 33 people consisting of 22 men with androgenic alopecia and 11 man who did not.

\subsection{Method of Collecting Data}

Patients who met the inclusion criteria and are willing to sign the informed concent, then he asked about his history and do a physical examination of hair and determine the grading classification of Norwood- Hamilton modification for man. Hair samples were taken in the area of the occipital 2-5 strands and then stored in a plastic container and sealed (free of dust, sweat, scaly epidermis, detergents and cosmetics) and then taken to the UNHAS chemical laboratory for analysis. Hair samples were then washed with water and dried, after drying, then transferred into a tube filled with tetrafluoretylen. Then add a solution of $\mathrm{HNO}_{3}$ and oxygenated water with a ratio 3: 1 . After 24 hours, we use microwave to dilute the minerals in hair samples and cool it, when cooled, we add $10 \mathrm{ml}$ volume samples using DW. Then the sample is inserted into the appliance atomic absorbance spectrophotometry then the results can be read. All procedures are performed in a clean condition. The data is collected and recorded and processed with statistical data using SPSS system.

\subsection{Data Analysis Technique}

The data we obtained were analyzed by SPSS. We do the descriptive analysis and the distribution of the hair zinc level. To know the difference between hair zinc levels in alopecia and not alopecia, weare using independent t- test if hair zinc levels distribute normally; if did not distribute normally, we used the Mann Whitney U test. To determine the relationship (correlation) between zinc levels in alopecia with Spearman correlation test. The significance used was $\alpha=5 \%$. The results of the analysis are collected in the form of a narrative, incorporating by tables or graphs.

\section{Result}

Studies have been conducted by using observational study design to determine hair and blood zinc levels in androgenic alopecia compared with controls. The study was conducted in Dermatology Department, Dr. Wahidin Sudirohusodo Hospital and Network Hospitals held on September-November 2015.

In alopecia group range of age between 25-51 years old with a mean 39.2 years, while in the control group, range of age between 21- 46 years with a mean 30.4 years; the results of the analysis independent $\mathrm{t}$ test showed a significant difference $(p<0.05)$. Patient with alopecia has older age than the control. BMI in both groups was not significant $(p>$ 0.05 ); BMI in alopecia groupbetween 18.1 to 25.0 with a mean of 23.05 , while the control ranged from 18.3 to 25.0 
with a mean of $22.41 \mathrm{~kg} / \mathrm{m}^{2}$. The Hamilton degree in

$1)$. alopecia group ranged between 1-7 with a mean of 2.9 (Table

Table 1. Characteristics of Study Sample.

\begin{tabular}{llll}
\hline \multirow{2}{*}{ Variable } & Min-Max/ Mean (SD) & & Pontrol $(\mathbf{n}=\mathbf{1 1})$ \\
\cline { 2 - 4 } & Alopecia $(\mathbf{n}=\mathbf{2 2})$ & $21-46 / 30,4(7,1)$ & $0,003^{*}$ \\
Age $($ years old) & $25-51 / 39,2(7,7)$ & $18,3-25,0 / 22,41(2,64)$ & $0,448^{*}$ \\
BMI $\left(\mathrm{kg} / \mathrm{m}^{2}\right)$ & $18,1-25,0 / 23,05(2,06)$ & - & \\
Hamilton Degree & $1-7 / 2,9(1,7)$ & & \\
\hline
\end{tabular}

*Independent $t$ test

The mean zinc levels lower in alopecia group (216.56 g) than the control $(272.61 \mathrm{~g})$, but the results of the analysis Mann Whitney test showed $\mathrm{p}=0.058$. When data tranformaed to be logged zinc levels; Independent test results showed a significant difference $(\mathrm{p}<0.05)$ which is lower the alopecia group(2.27) than control (2.42) (Table 2).

Table 2. Comparison Hair Zinc Level between Two Groups.

\begin{tabular}{llll}
\hline \multirow{2}{*}{ Variable } & \multicolumn{2}{l}{ Mean $(\mathbf{S D})$} & \multirow{2}{*}{ P } \\
\cline { 2 - 3 } & Alopecia $(\mathbf{n}=\mathbf{2 2})$ & Control $(\mathbf{n}=\mathbf{1 1})$ & \\
\hline Zinc Level $(\mu \mathrm{g} / \mathrm{gr})$ & $216,56(123,23)$ & $272,61(88,63)$ & $0,058^{* *}$ \\
Log Zinc level & $2,27(0,24)$ & $2,42(0,13)$ & $0,034^{*}$ \\
\hline
\end{tabular}

*Independent $\mathrm{t}$ test **Mann Whitey $\mathrm{U}$ test

To assess the comparison between the blood zinc level between alopecia and control groups, we do Independent $t$ test analysis zinc levels in two groups that distribute normally; besides transformation the data of zinc level to form logritma and after having tested in both groups that distribute normally, so the analysis of Independent $t$ test is to assess the differences between the two groups. Based on this comparison, there was no significant difference in blood zinc levels ( $p>0.05$ ) between two groups; nor may log blood zinc levels (Table 3 ).

Tabel 3. Comparison Blood Zinc Level between Two Groups.

\begin{tabular}{llll}
\hline \multirow{2}{*}{ Variable } & \multicolumn{2}{l}{ Mean (SD) } & p \\
\cline { 2 - 3 } & $\mathbf{A}(\mathbf{n}=\mathbf{2 2})$ & $\mathbf{B}(\mathbf{n}=\mathbf{1 1})$ & $0,986^{*}$ \\
\hline Zinc Level(mg/L) & $9,71(1,92)$ & $9,69(2,32)$ & $0,919^{*}$ \\
\hline Log Zinc level & $0,98(0,08)$ & $0,98(0,10)$ & . \\
\hline
\end{tabular}

*Independent t test

To assess factors associated with high hair zinc levels, to test the correlation between the blood zinc levels, age, BMI and Hamilton degrees. It was found that only the degree of Hamilton was correlated significant $(p<0.05)$ with high hair zinc levels. The higher the degree of Hamilton, the lower hair zinc levels. Blood zinc levels, age and BMI did not correlate significantly ( $\mathrm{p}>0.05)$ with high hair zinc levels (Table 4).

Tabel 4. Correlation between Blood Zinc Level, Age, BMI, and Hamilton Degree with Hair Zinc Level.

\begin{tabular}{llll}
\hline \multirow{2}{*}{ Variable } & \multicolumn{3}{l}{ Hair Zinc Level } \\
\cline { 2 - 4 } & $\mathbf{N}$ & $\mathbf{R}$ & $\mathbf{P}$ \\
\hline Blood Zinc Level & 33 & 0,126 & $0,242^{* *}$ \\
Age & 33 & 0,195 & $0,138^{* *}$ \\
BMI & 33 & 0,207 & $0,123^{* *}$ \\
Hamilton Degree & 22 & 0,395 & $0,034^{*}$ \\
\hline
\end{tabular}

*Pearson Correlation **Spearman Correlation

Based on the results of the study, we found that there is a significance pure correlation $(p<0.005)$ between the degree of Hamilton and age with $r=0,464$ and between hair zinc levels and Hamilton degrees with $r=0,405$, but it was not found significant correlation between age and hair zinc levels; where $r=0,125$ ( $\mathrm{p}>0.05)$ (Table 5).

Table 5. Bivariate and Partial Correction between Age, Hair Zinc Level, and Hamilton Degree.

\begin{tabular}{lllll}
\hline \multirow{2}{*}{ Variable } & \multicolumn{2}{l}{ Bivariate level } & \multicolumn{2}{l}{ Partial level } \\
\cline { 2 - 5 } & $\mathbf{R}$ & $\mathbf{P}$ & $\mathbf{r}$ & $\mathbf{p}$ \\
\hline $\begin{array}{l}\text { Hamilton degree and } \\
\text { hair zinc level }\end{array}$ & 0,299 & 0,088 & 0,405 & 0,034 \\
$\begin{array}{l}\text { Hamilton degree and } \\
\text { age }\end{array}$ & 0,465 & 0,015 & 0,464 & 0,017 \\
Hair zinc level and age & 0,195 & 0,138 & 0,125 & 0,294 \\
\hline
\end{tabular}

\section{Discussion}

This study shows that there are significant differences ( $p$ $<0.05$ ) between the hair zinc levels in androgenic alopecia and control. On blood zinc levels, we found no significant differences between androgenic alopecia and control. Hair zinc levels had a significant relationship with the degree of Hamilton, where the lower the hair zinc levels in androgenic alopecia, the greater the degree of Hamilton. However, we found different things between blood zinc levels and hair zinc levels with the degrees of Hamilton that there is no significant difference. In this study, we also found association between age and the degree of Hamilton, where age increase, the greater the degree of Hamilton.

Androgenic alopecia is a hair loss disorder characterized by progressive miniaturization of the hair follicle with typical gender-specific pattern. In humans, hair loss on the head is not life-threatening, but can adversely affect the quality of life the individual. Androgenic alopecia is mediated by genetic factors and androgen system. However, clinical practice shows that environmental factors also have an important role in the pathogenesis of alopecia. Integrity hair function largely depends on good and balanced nutrition. The influence of nutrition on the human causes the disease scale that result in excessive hair loss. Therefore, it is important to separate the nutritional factors that act directly on the skin from those who act only on hair follicles. [8]

Zinc is involved in stabilizing cell membranes and prevents oxidative damage caused by free radicals. Zinc is a 
major structural component of a large number proteins with specific functions. Tracing elements such as copper and zinc are especially required to the function in the carbonic anhydrase enzyme, superoxide dismutase, polymerase, and collagenase as well as cell division, metabolism of nucleic acids and coenzyme. So, it takes about 300 enzyme functions. Also, zinc and copper play an outstanding role in the activity of antioxidant enzymes, and their value can serve as a surrogate marker of enzyme activity. Zinc deficiency can cause recurrent infections, hypogonadism, weight loss, mental disorders, eczema, alopecia, night blindness, loss of appetite, and delayed wound healing. The mechanism of how zinc affect the hair follicles is unknown. But the common belief, that zinc is also known element associated with the release of the hair and a deficiency of zinc can cause hair loss. [8]

In this study, the sample characteristics such as age and the incidence of androgenic alopecia showed that in the alopecia group, agebetween 25-51 years with mean 39.2 years, while in the control group, age between 21- 46 years with mean 30.4 years; the results of the analysis of independent $t$ test showed a significant difference $(p<0.05)$. Age in alopecia group are older than the control. This is consistent with study by Shankar et al (2009), conducted in India with the results based on age at onset androgenic alopecia commonly found in males is in third decade $(34.4 \%)$. Several studies have reported the highest incidence of androgenic alopecia about $96 \%$ in Caucasian racemale. With $62 \%$ age 20 to 40 years old have bitemporal recession, and $54 \%$ of Caucasian race male whose more than 30 years old have experienced androgenic alopecia. [1, 9]

BMI in alopecia group obtained higher scores than the control but not significant ( $p>0.05)$. Where the BMI in alopecia group are between 18.1 to 25.0 with a mean of 23.05, while the control ranged from 18.3 to 25.0 with a mean of $22.41 \mathrm{~kg} / \mathrm{m}^{2}$. This is according to the study by Otzurk et al (2014), which was done in Turkey to get the BMI of alopecia patients was higher than control, but in that study, they showed significant $(p<0.05)$. In that study, reported that the increasing BMI in patients with androgenic alopecia can cause an imbalance of nutrients that play a role in the pathogenesis of androgenic alopecia. [8, 10]

The mean of hair zinc level is lower in alopecia group $(216.56 \mathrm{pg} / \mathrm{g})$ than control $(272.61 \mathrm{pg} / \mathrm{g})$, but the results of the analysis Mann Whitney test showed $p=0.058$. When data transformed to be logged Zinc levels; Independent test results showed a significant difference $(\mathrm{p}<0.05)$ in Alopecia group is lower (2.27) than control (2.42). It is also consistent with studies in Turkey which showed hair zinc level $(0.03)$ were significantly lower in patients with alopecia compared with control (0.42). In the study by Otzurk et al (2014), reported that hair zinc levels may have a role in etiopathogenesis and treatment of androgenic alopecia. Most of biochemical functions require zinc, such as immune activity, protein synthesis, and hormone activity. Zinc also has an important role in physiology and biochemistry. However, its role in hair follicle is still unclear, since there are only a few studies on hair zinc level in patients with androgenic alopecia. [8]

The comparison between blood zinc level in alopecia and control groups, in analysis Independent t test for the zinc levels of the two groups distribute normally; besides the data transformation of zinc level to logarithm form and after having tested in both groups, the distribute normally, and we do analysis of Independent $t$ test to assess the differences between two groups. There is no significant difference in blood zinc levels ( $p>0.05$ ) between alopecia and control group; nor log levels of blood zinc. In the study by Otzurk et al (2014) also did not show any significant differences in serum zinc levels and urine in androgenic alopecia patients compared with control. [8]

Only Hamilton degree were correlated significantly ( $p$ $<0.05)$ with high hair zinc levels. The higher the degree of Hamilton, the lower hair zinc levels. The relationship between the hair zinc levels with the degree of alopecia doesn't have definite explanation, but if it is correlated with the age in which the degree of alopecia and age are positively correlated. As the research conducted by Shankar et al (2009), that conducted in India, the study reported an increased prevalence of androgenic alopecia with their age. The degree of alopecia increased from the initial stage I and II become more severe following the age. [9]

Based on this study, we found a correlation between the degree of Hamilton with age, whereas the older the age, the higher Hamilton degree from alopecia patients. Where the study in India by Shankar et al (2009), also reported that the prevalence of androgenic alopecia increases with age. Hamilton degrees increased from mild to severe stages along with age. There is also a relationship between the degree of Hamilton and hair zinc level, where the higher the degree of Hamilton, the lower hair zinc levels. A correlation between hair zinc levels and androgenic alopecia is also found in study conducted by Otzurk et al (2014), which found that there is a low hair zinc levels in patients with androgenic alopecia, but for its relationship to the degree of Hamilton is still unknown in the study. $[8,9]$

From the results of this study, we can find that the hair zinc levels may play a role in the occurrence of androgenic alopecia. Where the hair zinc levels decreased in patients with androgenic alopecia. When dealing with hair loss, zinc is a potent inhibitor of a regression of hair follicles and accelerate the recovery of the hair follicle. Zinc also cause modulation of the activity of type 1 and $25 \alpha$ reductase. [11, 12]

Study by Otberg et al (2007) reported that the prevalence increases with age. Where $53 \%$ of men who age $40-49$ years showed hair loss symptoms is moderate until severe according to the Hamilton degree grade 3 or more. In this study, we also found Hamilton degree influenced by age. Where the older patients with androgenic alopecia, the greater the degree of Hamilton. [4]

\section{Conclusion}


Researchers concluded that there is a difference hair zinc levels between alopecia and control, but there are no differences in blood zinc levels. Hair zinc levels were lower in control than alopecia. There is an association between hair zinc levels with the degree of Hamilton, where the lower hair zinc levels in patients with alopecia, the greater the degree of Hamilton. But blood zinc levels are not associated with hair zinc level as well as the degree of Hamilton. It was also found the relationship between age and the degree of Hamilton. The older age, the greater the degree of Hamilton. However, no relationship was found between age and hair zinc levels. Similarly, between BMI with the degree of Hamilton. There is a relationship between BMI and androgenic alopecia, whereas the IMT in patients with alopecia is lower than in the control. But it is not significantly different. Researchers suggest that further study onzinc topical treatment in the hair to androgenic alopecia patients, especially in younger patients and other associated factors with the occurrence of androgenic alopecia is needed.

\section{Refferences}

[1] Otberg N, Finner AM, Shapiro J. Androgenetic Alopecia. Endrocinol Metab Clin N Am 2007; 36: 379-98.

[2] Stough D, Stenn K, Haber R, Parsley W, Vogel J, Whiting D. Psychological Effect, Pathophysiology, and Management of Androgenetic Alopecia in Men. Mayo Clin Proceeding 2005; 80 (10): 1316-22.

[3] Hunt N, Mchale S. The Psychological impact of alopecia. Journal of Personality and Sosial Psychology 2007; 20: 362-4.
[4] Otberg N, Shapiro J. Hair Growth Disorders. In: Goldsmith LA, Katz SI, Gilchrest BA, Paller AS, Leffell DJ, Klauswolff, editors. Fitzpatrick's Dermatology in General Medicine. 8th ed. New York: McGraw Hill; 2012. p. 1374-85.

[5] Gupta M, Mysore V. Classification of Patterned Hair Loss: A Review. J Cutan Aesthet Surg 2016; 9: 3-12.

[6] Hamad WAM, Said AF, Hamid AA. Role of Some Trace Elements in the Pathogenesis of Telogen Effluvium in Egyptian Females. J Egypt Women Dermatol Soc 2010; 7 (1): 44-8.

[7] Kil MS, Kim CW, Kim SS. Analysis of Serum Zinc and Copper Concentrations in Hair Loss. Ann Dermatol 2013; 25 (4): 405-9.

[8] Otzurk P, Kurutas E, Ataseven A, Dokur N, Gumusalan Y, Gorur A. BMI and levels of zinc, copper in hair, serum and urine of Turkish male patients with and rogenetic alopecia. $J$ of Trace Elements in Med and Bio 2014; 28: 266-70.

[9] Shankar K, Chakravarthi M, Shilpakar R. Male Androgenetic Alopecia: Population Based Study in 1.005 Subjects. Int $J$ Trichology 2009; 1 (2): 131-3.

[10] Ellis J, Sinclair R, Harrap S. And rogenetic alopecia: pathogenesis and potential for therapy. $J$ Invest Dermatol 2009: 1-11.

[11] Dastgheib L, Mostafavi Z, Adnan A, Khoshdel Z, Sadat M, Ahrari I, et al. Comparison of $\mathrm{Zn}, \mathrm{Cu}$, and $\mathrm{Fe}$ Content in Hair and Serum in Alopecia Areata Patients with Normal Group. Dermatology Research and Practice 2014: 1-5.

[12] Gupta M, Mahajan VK, Mehta KS, Chauhan PS. Zinc Therapy in Dermatology: A Review. Dermatology Research and Practice 2014: 1-11. 